\title{
Hybrid Integration of Graphene Analog and Silicon Complementary Metal-Oxide-Semiconductor Digital Circuits
}

\author{
Seul Ki Hong ${ }^{1}$, Choong Sun Kim ${ }^{1}$, Wan Sik Hwang ${ }^{2}$ and Byung Jin Cho ${ }^{1, *}$ \\ ${ }^{1}$ Department of Electrical Engineering, KAIST, Daejeon, 305-701, Republic of Korea \\ ${ }^{2}$ Department of Materials Engineering, Korea Aerospace University, Gyeonggi-do, 412-791, Korea,
}

To whom correspondence should be addressed. "E-mail: bjcho@kaist.edu

1. Process flow

2. Characterization of MOSFET and ring oscillator

3. Characterization of graphene circuit 


\section{Process flow}

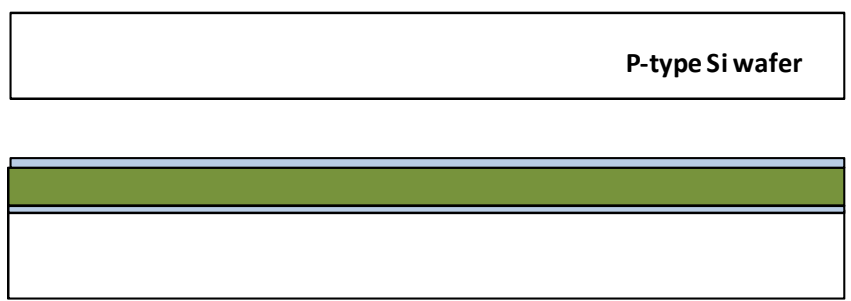

Substrate prepare

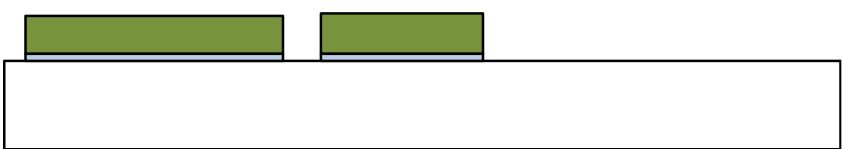

Masking oxide / $\mathrm{Si}_{3} \mathrm{~N}_{4} /$ buffer oxide deposition

Active area definition

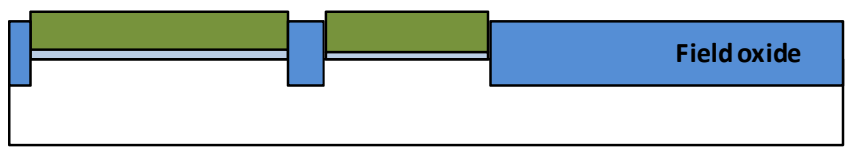

Field oxidation (LOCOS)

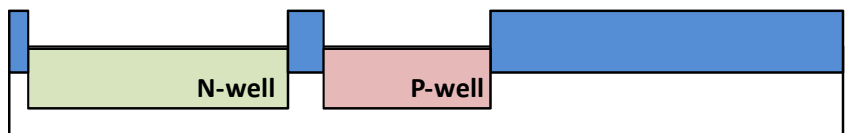

P-well \& N-well definition

(Boron \& Phosphorus implant and drive)

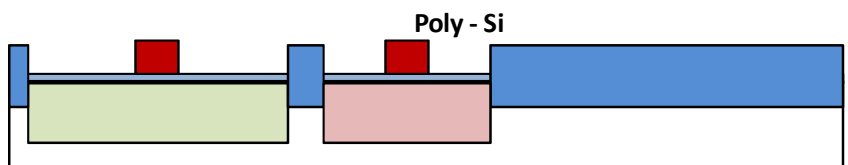

Gate oxidation (300 A)

Poly-Si gate deposition by LPCVD

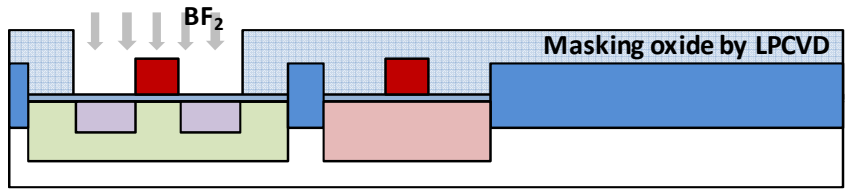

Active NMOS shield

$\mathrm{BF}_{2}$ implant for PMOS source / drain formation

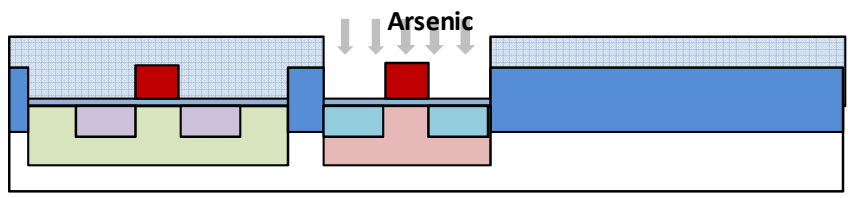

Active PMOS shield

Arsenic implant for NMOS source / drain formation

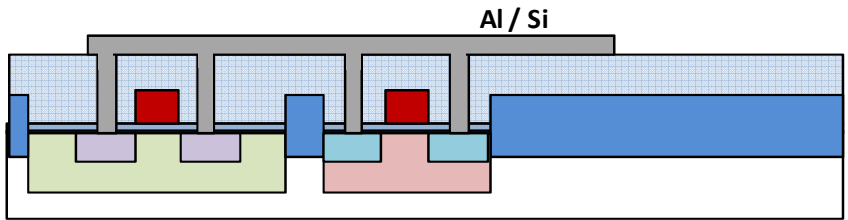

$\mathrm{SiO}_{2}$ deposition for passivation

Al / Si metallization by sputtering

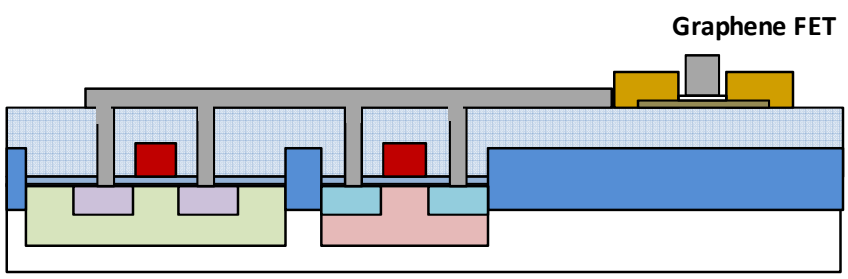

Transistor based graphene on passivation layer fabricated

Figure S1. Graphene and CMOS hybrid-integrated circuit fabrication process summary. 


\section{Characterization of MOSFET}

a) $10^{\circ}$
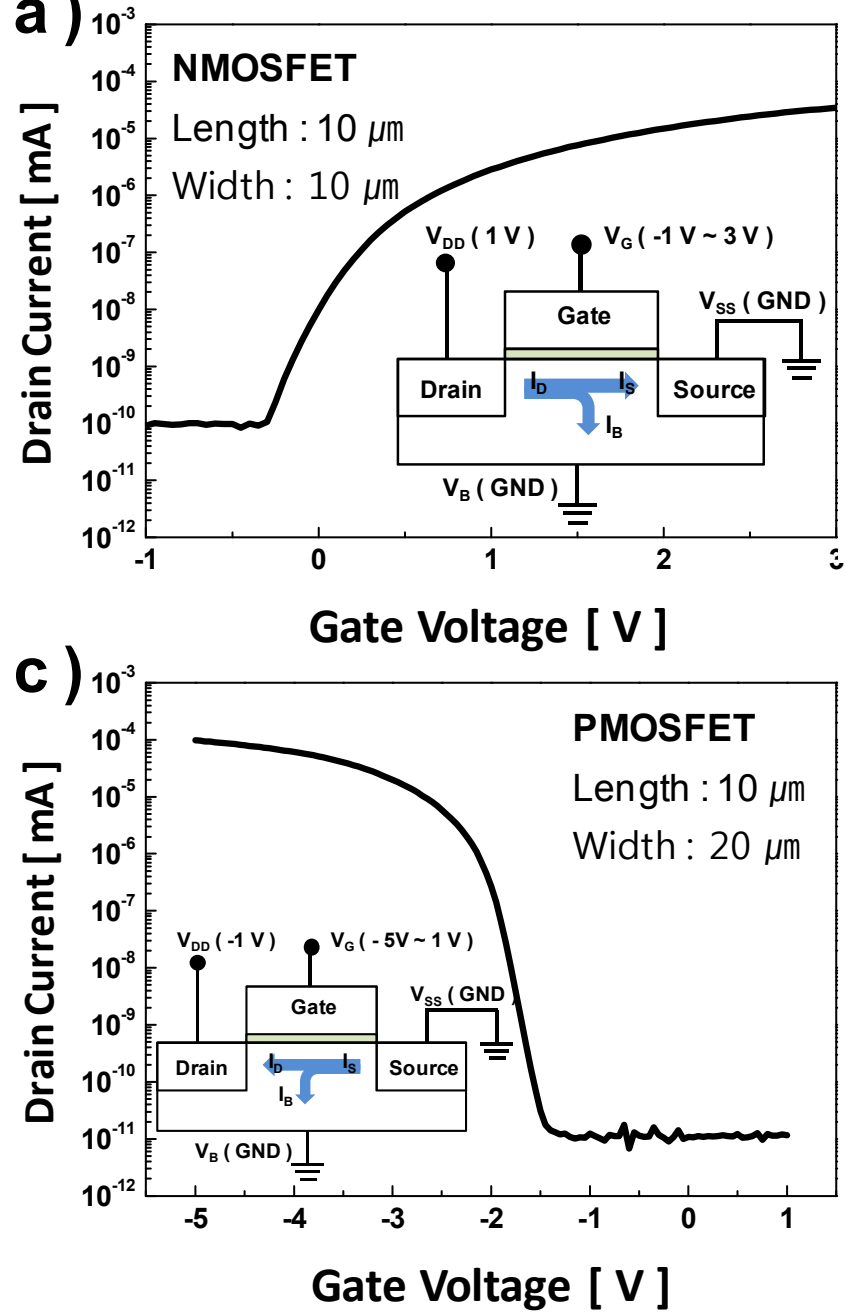

b )
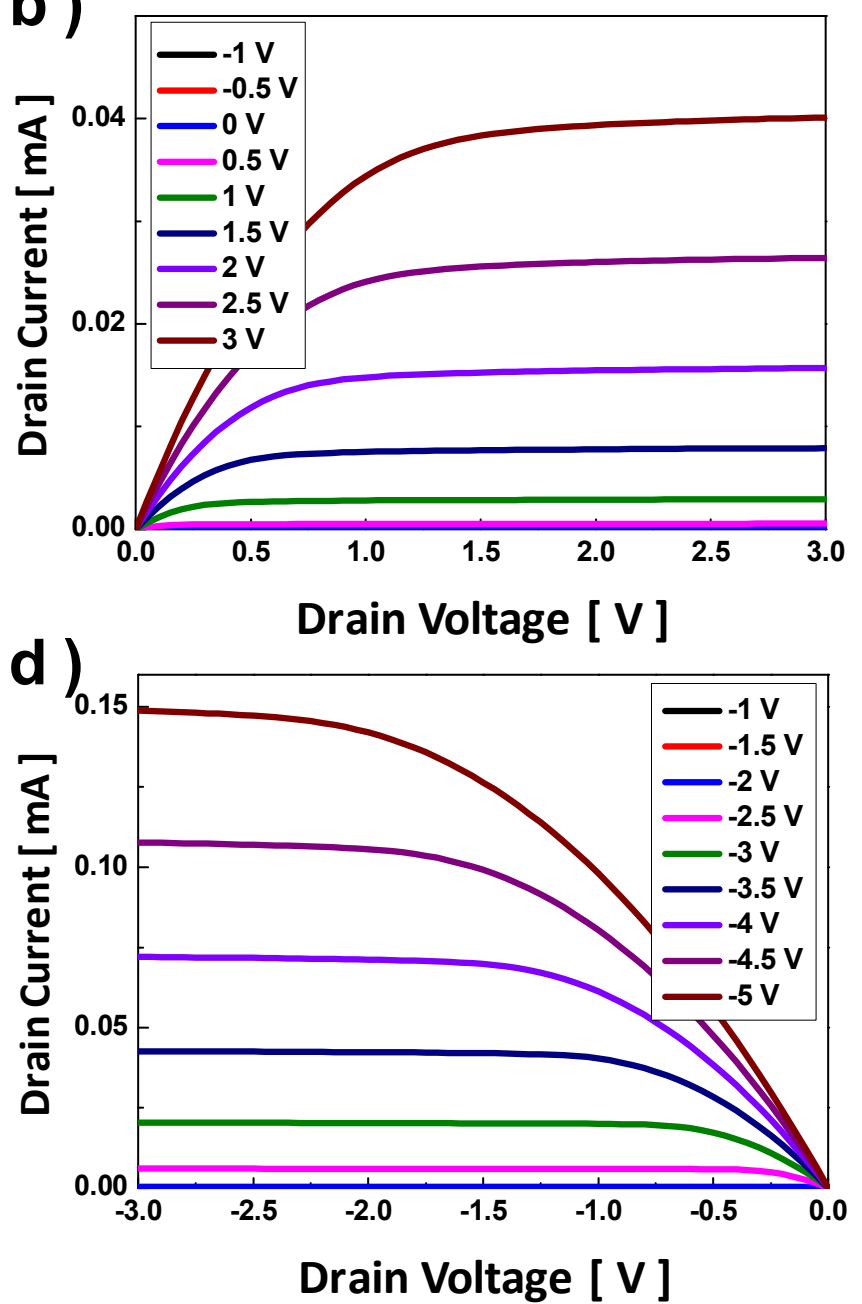

Figure S2. Electrical properties of NMOS and PMOS transistors. (a) $I_{D}-V_{G}$ characteristics at $V_{D}=1 V$, and (d) $I_{D}-V_{D}$ characteristics at $V_{G}=-1 V$ to $3 \mathrm{~V}$ of the NMOS transistor. (c) $I_{D}-V_{G}$ characteristics at $V_{D}$ $=-1 \mathrm{~V}$, and (d) $\mathrm{I}_{\mathrm{D}}-\mathrm{V}_{\mathrm{D}}$ characteristics at $\mathrm{V}_{\mathrm{G}}=-1 \mathrm{~V}$ to $-5 \mathrm{~V}$ of the PMOS transistor. 
a)

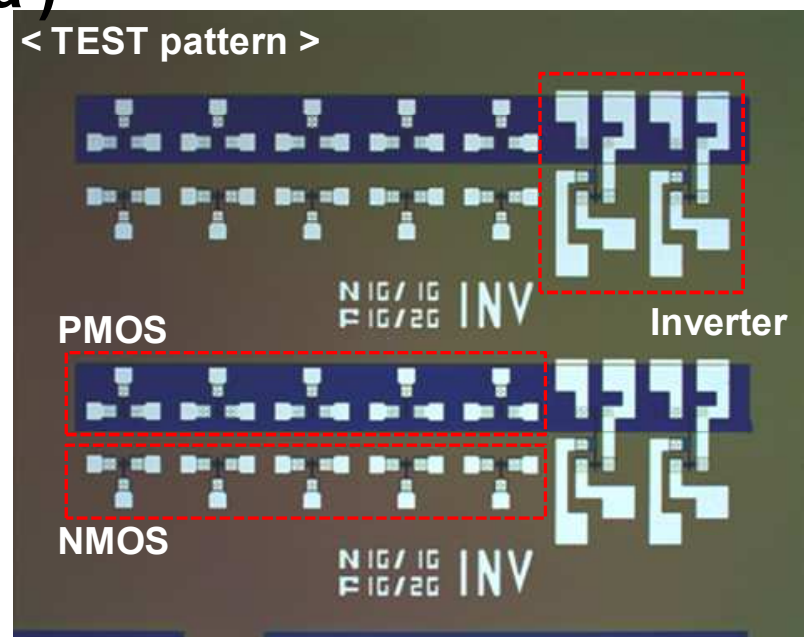

b)

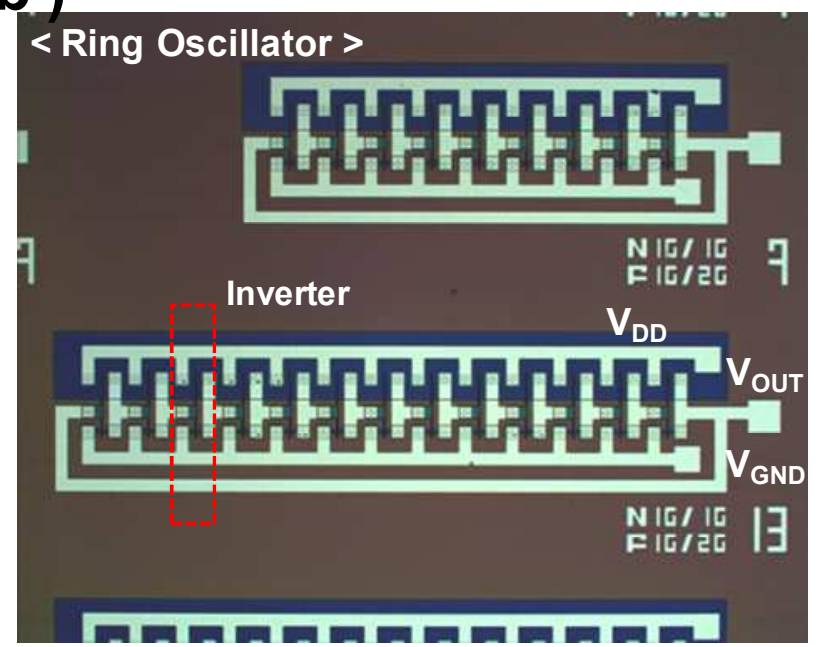

Figure S3. Optical microscope image of CMOS based transistor and ring oscillator. (a) NMOS and

PMOS transistor to confirm the electrical properties of unit transistor. (b) Image of 9 inverting stage and 13 inverting stage ring oscillator. 

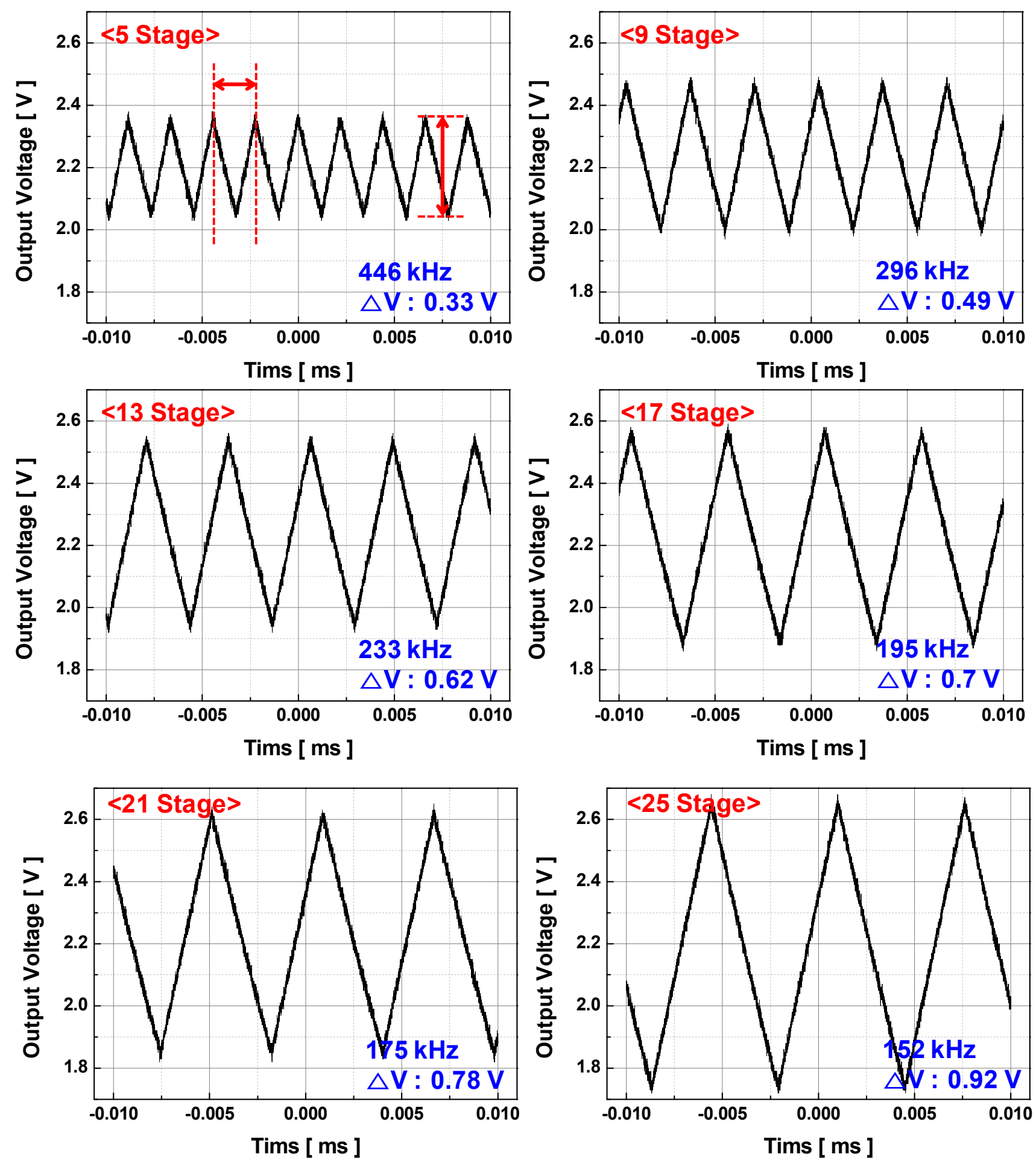

Figure S4. Output voltages depending on various inverting stages of CMOS based ring oscillator. The frequency and peak-to-peak voltage has been changed by number of stage in ring oscillator 


\section{Characterization of graphene circuit}
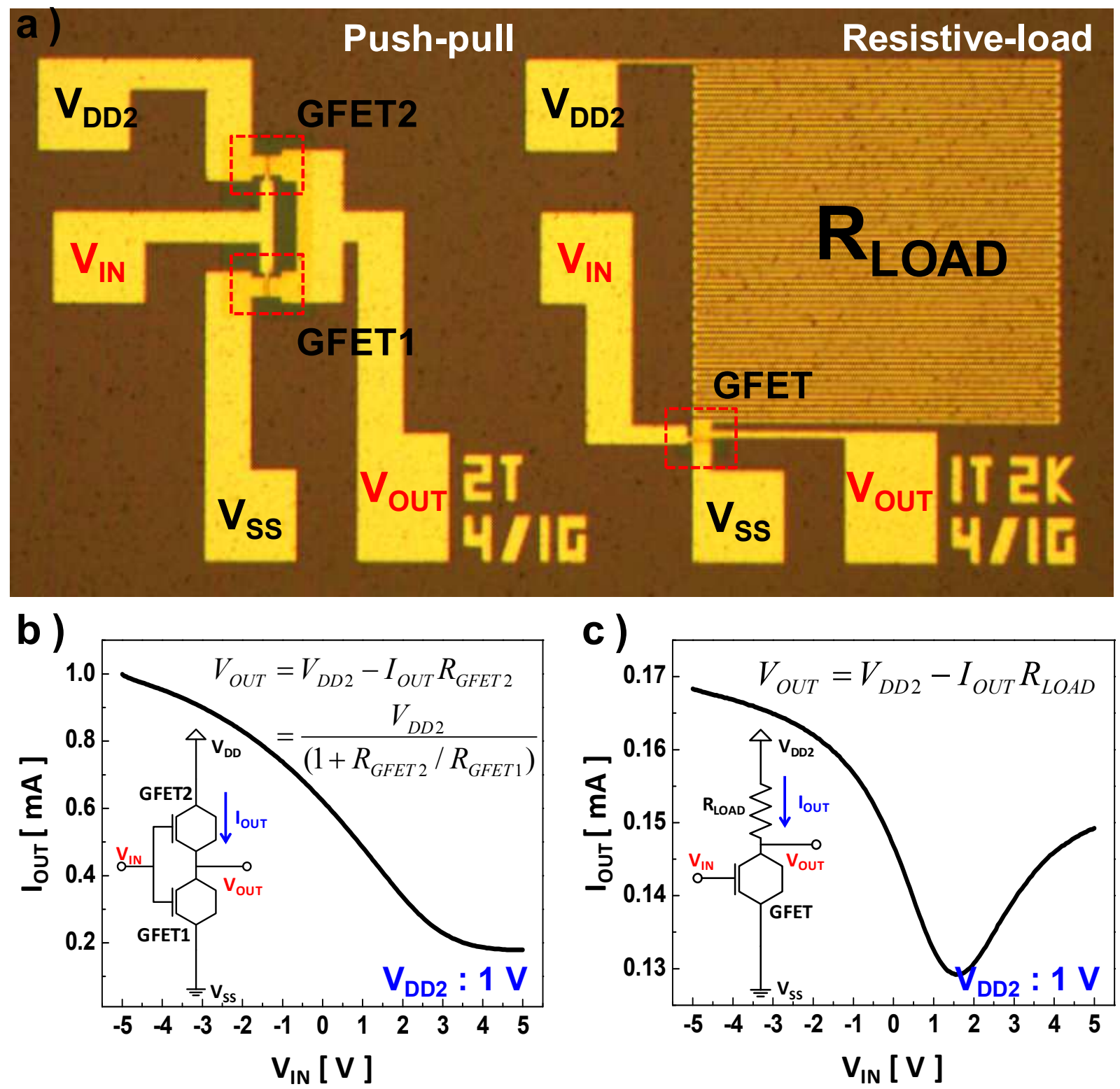

Figure S5. (a) Optical microscope image of graphene based multi-mode phase shifters, which are fabricated with both in complementary push-pull and resistive-load structure. (b) I IOUT-V $\mathrm{V}_{\text {IN }}$ characteristics at $\mathrm{V}_{\mathrm{DD} 2}=1 \mathrm{~V}$ of the push-pull structure and (c) resistive-load structure.

Multi-mode graphene phase shifters were realized both in a complementary push-pull and resistive-load structure. In push-pull structure, the potential of GFET2 is higher than GFET1 when the $\mathrm{V}_{\mathrm{DD} 2}$ is applied, 
which therefore make difference between dirac voltage of GFET2 and GFET1. This complementary push-pull structure is considered to be integrated since GFET2 loads GFET1, and both are fabricated on the same graphene layer. It does not require any additional load for circuit operation. The resistive-load structure is obtained by replacing GFET2 with load resistor. The voltage gain is depending on dirac voltage of GFET. The operating voltage of the graphene FETs (GFETs) was set to $1 \mathrm{~V}$, because of the high current density of GFET due to extremely thin graphene channel layer. The higher operating voltage in the GFET can result in too high current density which can cause device reliability issue due to excessive Joule heating of the graphene device. On the other hand, the operating voltage of the Si ICs was set to $5 \mathrm{~V}$, because the silicon FETs used in this work are long channel devices due to the process facility limitation of university fab. It is worthy to note that modern commercialized silicon CMOS circuits use sub-0.1 $\mu \mathrm{m}$ design rule and the power supply voltage is around $1 \mathrm{~V}$. If graphene FETs are integrated with the advanced silicon CMOS devices, the operating voltage will be no more an issue. 